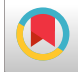

\title{
Spectrum of Mutations of Familial Mediterranean Fever Gene by Whole Sequencing Method in Iranian Patients
}

\author{
Helal Nemat Farahzadi (iD ${ }^{1,2}$, Mohammad Taghi Akbari (iD) ${ }^{1}$, Reza Shiari (iD ${ }^{3,}{ }^{*}$, Shohre Zare Karizi (iD ${ }^{4}$ and \\ Shirin Farivar (ib) ${ }^{5}$ \\ ${ }^{1}$ Tehran Medical Genetics Laboratory, Tehran, Iran \\ ${ }^{2}$ Department of Biological sciences, Technical and Vocational University (TVU), Tehran, Iran \\ ${ }^{3}$ Department of Pediatric Rheumatology, Mofid Children's Hospital, Shahid Beheshti University of Medical Sciences, Tehran, Iran \\ ${ }^{4}$ Department of Biology, Varamin Pishva Branch, Islamic Azad University, Tehran, Iran \\ ${ }^{5}$ Cell and Molecular Biology Department, Faculty of Life Sciences and Biotechnology, Shahid Beheshti University, Tehran, Iran \\ "Corresponding author: Department of Pediatric Rheumatology, Mofid Children's Hospital, Shahid Beheshti University of Medical Sciences, Tehran, Iran. Email: \\ shiareza@yahoo.com
}

Received 2020 November 15; Accepted 2021 September 28.

\begin{abstract}
Background: Familial Mediterranean fever (FMF) is the most common type of periodic fever syndrome. The disease is most prevalent in the western Mediterranean population, but today it is widespread in the world due to the large ethnic migrations of Turks, Jews, Arabs and Armenians. The MEFV gene is the only gene known to be associated with the disease.

Objectives: The aim of this study was to characterize pathogenic mutations in patients with typical FMF symptoms by sequencing the entire MEFV gene.

Methods: This is a descriptive-analytical study that was performed during ten years from 2009 to 2019 . On 252 patients after clinical diagnosis based on existing criteria to determine mutations referred to Tehran Medical Genetics Laboratory and the whole sequencing method for MEFV gene was used to determine mutations.

Results: Out of 252 patients, 143 (56.7\%) had pathogenic variants, and 109 (43.3\%) had no variants reported as pathogenic mutations. Variants were identified as fallow: (1) $8.7 \%$ as homozygous; (2) $22.2 \%$ as compound heterozygous; (3) $25.7 \%$ as heterozygous. The most common variants were M694V (c.2080A $>$ G) and E148Q (c.442G>C).

Conclusions: This study showed that the age of onset of the disease was in the first and second decades of life amongst our patients and the most common complaints of patients were periodic fever and abdominal pain. The most frequent allele was M694V (c.2080A>G) followed by E148Q (c.442G>C) allele.
\end{abstract}

Keywords: Common Mutations, MEFV Gene, Autosomal Recessive, Iranian Population, Familial Mediterranean Fever

\section{Background}

Familial Mediterranean fever (FMF) is characterized by acute, self-limited episodes of fever and polyserositis recurring at irregular intervals $(1,2)$. Recent studies show that the symptoms of some FMF heterozygotes range from mild to classic. Symptoms of autosomal recessive heterozygous inheritance are altered by incomplete penetration or expression. It is characterized by fever and muscle aches, pleurisy, arthritis, or inflammation of the skin and subcutaneous tissue. The most important complication of FMF disease is the development of amyloidosis, which eventually leads to kidney failure. Symptoms of this disease can appear in the first 10 years of life and according to the latest statistics, more than $80 \%$ of patients show symptoms of this disease in childhood and adolescence.
Symptoms of recurrence include fever and severe abdominal pain that usually lasts one to three days and resolves spontaneously. The gene associated with this disease is the MEFV gene, which was first identified on the short arm of chromosome 16 (16p13.3) in 1997 and was independently characterized by spatial cloning by two working teams (35). By gene cloning, four mutations in exon 10 were identified as M694I (c.2082G>A), M694V (c.2080A>G), M680I (c.2040G >A), V726A (c.2177T>C). These four mutations and the $\mathrm{E} 148 \mathrm{Q}$ (c.442G $>\mathrm{C}$ ) mutation in exon 2 are the most common of the 59 hypothetical mutations identified so far that are thought to be associated with the disease $(3,4)$. Among them, M694V (c.2080A>G) mutation has the highest frequency in connection with acute FMF disease. However, it should be noted that incomplete penetration, alteration of MEFV gene expression and the possibility of the presence

Copyright (c) 2021, Journal of Human Genetics and Genomics. This is an open-access article distributed under the terms of the Creative Commons 
of other possible genetic factors can affect the expression of this gene in patients $(6,7)$. The gene has 10 exons, it encodes 781-amino acid protein called Pyrin or Marenostrin with a molecular weight of 86,000 daltons. This protein is mainly expressed in granulocytes and is thought to be involved in apoptosis $(3,4)$.

Pyrin also plays a negative regulatory role in the body's inflammatory reactions. The initial diagnosis of FMF is mainly based on clinical manifestations, ethnicity, family history, and response to colchicine (8). While the definitive diagnosis of this disease is determined by examining the MEFV gene and identifying mutations in the exons of the MEFV gene. Mutations in the MEFV gene are detectable in $50-80 \%$ of cases, but not all existing mutations are currently detectable and there are known and common mutations in $80 \%$ of patients. Although the clinical signs of the disease are specific and typical, it is important to determine the type of mutation to diagnose the disease. However, it should be noted that the absence of mutations in the MEFV gene does not rule out the possibility of disease (9). To date, more than 90 diseaserelated mutations have been identified, but a total of 389 variant sequences have been identified in the FMF gene (http://www.fmf.igh.cnrs.fr/ISSAID/infevers/search.php). Because the MEFV gene is the only gene associated with the disease, it was studied in this study for possible mutations.

\section{Objectives}

Our aim was to investigate the spectrum and distribution of MEFV mutations in Iranian patients using a comprehensive method of mutation identification.

\section{Methods}

During a period from 2009 to 2019, 252 patients with symptoms similar to those of FMF were referred to Tehran Medical Genetics Laboratory (Akbari) by rheumatology and internist specialists. In most of these patients, symptoms of fever, periodic abdominal pain, and inflammation of the pleura, swelling of the joints, muscle pain, diarrhea in children, and constipation in adults. Treatment was observed in response to colchicine. Information such as date of birth, race, sex, symptoms and family history were mentioned in the questionnaires prepared for each patient. Written consent was obtained from patients or their companions at the time of sampling. 5 cc of peripheral blood was collected in tubes containing EDTA as anticoagulant. Genomic DNA was extracted using the salting out method. Primers were designed for all exons of MEFV gene and then PCR conditions were optimized for sequencing with forward and reverse primers
(Table 1). After replicating the target regions, the sequences were compared with the Cambridge reference sequences available at the NCBI site. The types of polymorphisms and mutations were identified from the FMF Database (http://fmf.igh.cnrs.fr/ISSAID/infevers/).

\section{Results}

In this study, out of 252 patients who were referred, 140 (55.5\%) were male. The average age of men was 17.95 years and their age range were between 1 and 48 years. The average age of women was 17.3 years and their age range were between 2 and 63 years. The age of more than $63.5 \%$ of patients (160 patients) was under 20 years. In this study, periodic fever and abdominal pain were the most common clinical manifestations observed among patients. Out of 252 patients, 143 (56.7\%) had pathogenic variants, whereas in 109 (43.3\%) no reported pathogenic mutations were observed while these patients showed the clinical picture of FMF patients and responded well to the treatment. Variants were identified in $8.7 \%$ of the patients as homozygous, 22.2\% of patients with variant as compound heterozygous, and $25.7 \%$ of mutant patients as heterozygous (Table 2 ). Most variants were M694V (c.2080A>G), E148Q (c.442G>C) and R202Q (c.605G $>A$ ) (Figure 1), respectively. In a person with symptoms of fever, periodic abdominal pain, and constipation, an unreported mutation D308N (c.922G >A) was observed as a compound heterozygote with a G304R (c. 910G $>$ A) mutation (Figure 2).

\section{Discussion}

Our results show that common mutations consist of more than $30 \%$ of the identified alleles (Table 2). M694V (c.2080A $>\mathrm{G}$ ) is the most common mutation, accounting for about $27.3 \%$ of the mutations detectable in this study, with M694V/M694V (c.2080A>G)/(c.2080A>G) accounting for $4.4 \%$ of homozygous variants (Table 2 ), as reported in other studies (10-14). The E148Q (c.442G>C) variant then shows the highest frequency (Table 2), which is similar to that seen in Europeans (7). In each pathogenic variant form, there was no statistical difference in the relationship between clinical symptoms and type of mutation. The only notable point was the frequency of fevers in R202Q mutations, which, although more common than other mutations, was not statistically significant. Patients with the M694V compound heterozygous mutation have had more severe and sometimes refractory heartburn. In these patients, serum ferritin levels sometimes increased and protein was observed in the urine. Compared to the other FMF patients in our study, these patients required 


\begin{tabular}{lcc}
\hline Table 1. Sequence of Primers $\left(5^{\prime}-3^{\prime}\right)$ Used to Sequence All Exons of MEFV Gene & & \multicolumn{1}{c}{ Reverse } \\
\hline Exon & Forward & PCR.P $(\mathbf{b p})$ \\
\hline $\mathbf{1}$ & AGTCTGGAGGAGAACAGTTGAC & TCTCAAAGAACAGGTGTTCCAG \\
$\mathbf{2}$ & TTCTCTCTCCTCTGCCCTGAATC & CTCACTACATTCACCAGGCTGGTC \\
$\mathbf{3}$ & GAACTCGCACATCTCAGGCAAG & CCCAAGAATGCTGGTTAATGCAC \\
$\mathbf{4}$ & CTCTTTCCTGAGCACCCCTC & CCATCTTTCTGCAGTAGTCACC \\
$\mathbf{5 - 6}$ & GTTCCAGACAATATCGCCTCCTG & CTTAGCCCAGCTCCACTCCAAAC \\
$\mathbf{7 - 8 - 9}$ & GGTGTGCCCTGTGGAGAATGTAG & CGGAGAGCCCAGAACAATGATAC \\
$\mathbf{1 0}$ & GAACCCTGTAGGGATGTTGC & CTAGTCGGCATTCCGTGAC \\
\hline
\end{tabular}

${ }^{\mathrm{a}}$ The primer was designed so that it is displayed in sequencing with Forward exon 7 and 8 and Reverse exon 9 primer.

G

A

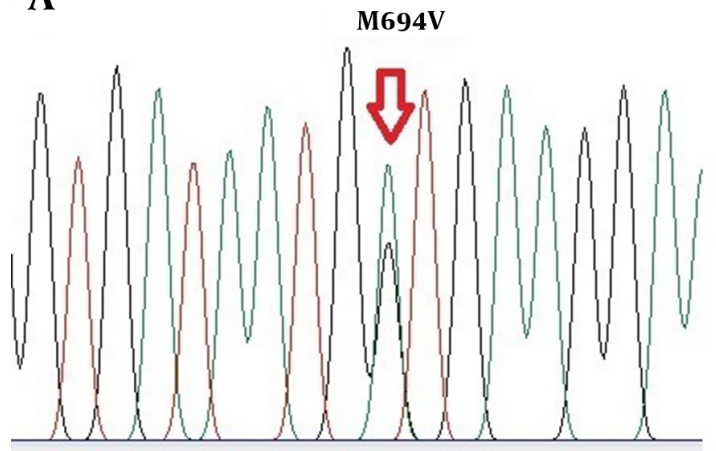

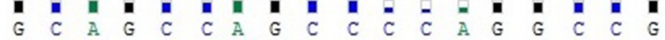

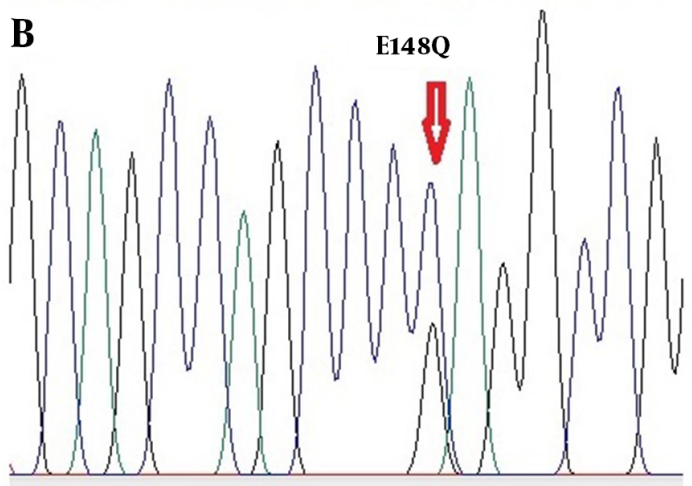

Figure 1. Electrogram; $A, M 694 V(c .2080 A>G)$ variant; B, E148Q (c.442G>C) variant

A

A. A G G

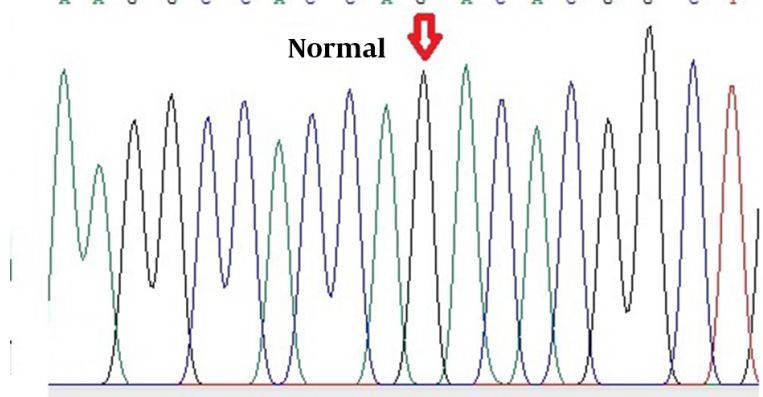

B

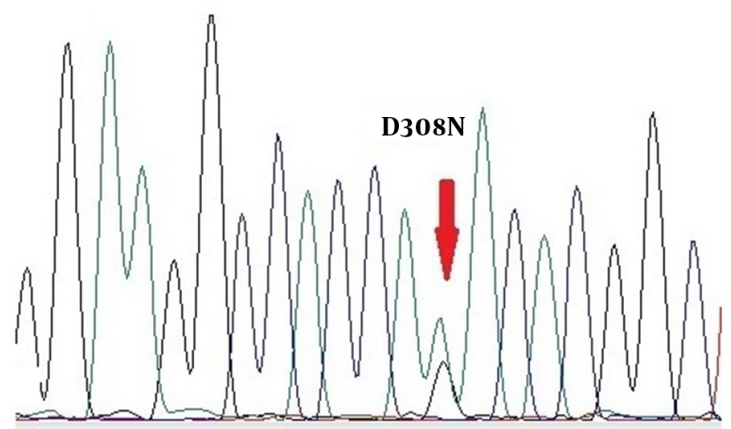

Figure 2. Electrogram of the new MEFV D308N variant (c.922G>A). A, wild type; B, G>A change in nucleotide 922 in exon 3, conversion of aspartic acid to asparagine changes to codon 308

higher doses of colchicine to control the disease and sometimes even continued use of steroids in addition to daily colchicine to maintain proteinuria. In 109 (43.3\%) no pathogenicity variants were observed, this may be due to various reasons, including the presence of rare and unknown mutations (in the promoter region, inside the introns or in untranslated region 3 ), genetic heterogeneity $(15,16)$ the presence of modifying genes $(17)$ and envi- 


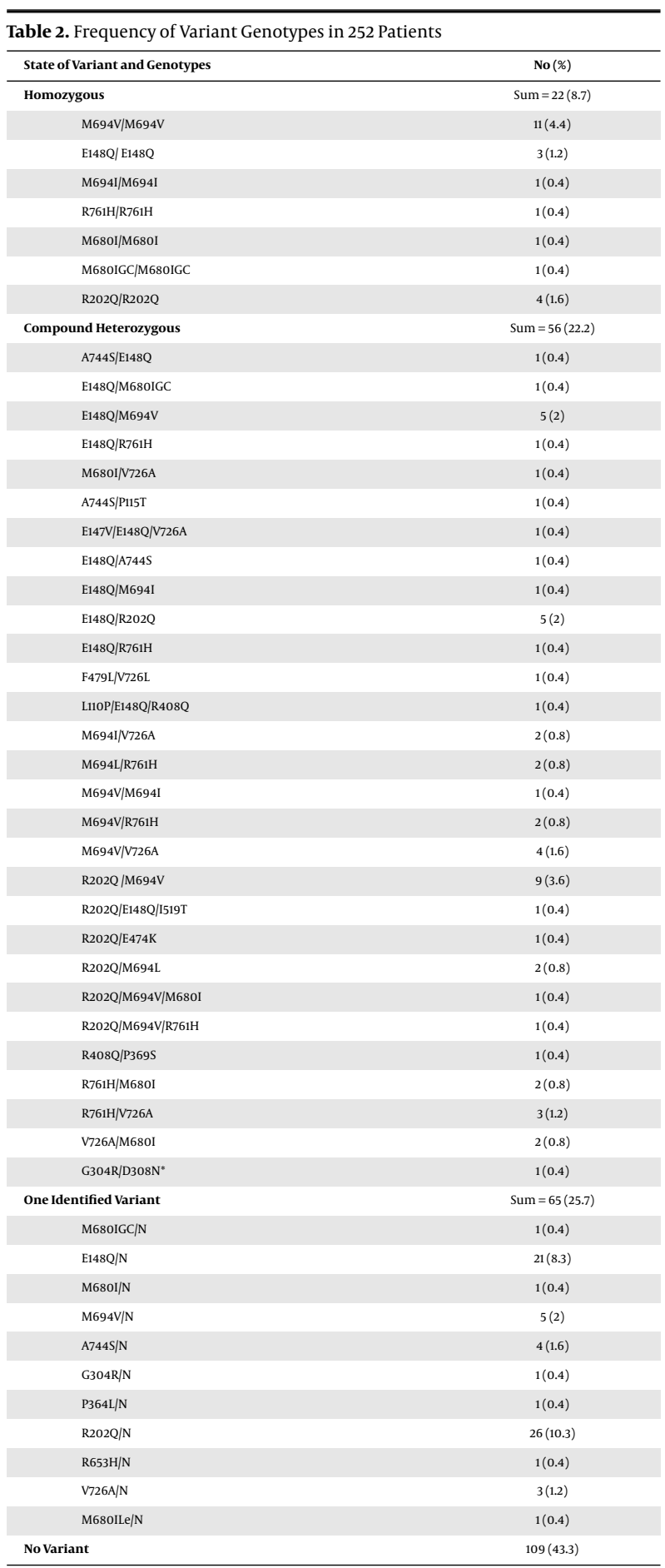

ronmental factors. Misdiagnosis with other hereditary recurrent febrile syndromes is also possible. It is involved in a relatively high percentage of non-mutant alleles (10). Molecular analysis of the MEFV gene in a patient with the variant D308N (c.922G >A) showed that the exon 3 gene was heterozygous. This variant replaces the amino acid aspartic acid with asparagine. The mutation R202Q (c.605G>A) had an overall frequency of about $10.3 \%$, and shows the highest frequency in the patient group with heterozygous variants. R202Q (c.605G $>$ A) was first identified in exon 2 in the MEFV gene by Bernot et al. (1998) who proposed this variant as a general polymorphism (2). In addition, in the FMF database, $\mathrm{R} 202 \mathrm{Q}$ (c.605G $>\mathrm{A}$ ) is shown as a polymorphism associated with linkage disequilibrium with M694V (http://www.fmf.igh.cnrs.fr/ISSAID/infevers/search.php).

According to this study, in our population, this mutation can be considered pathogenic as evaluated in Turkish FMF patients. There was a high association between MEFV gene R202Q polymorphism and FMF (18). In a study of 26 FMF patients in Greece, the R202Q (c.605G $>$ A) variant was found to be homozygous in 4 patients but was not observed in 60 healthy individuals. The change in $\mathrm{R} 202 \mathrm{Q}$ (c.605G $>$ A) may be more of a mutation than a polymorphism, and it has a dose-dependent effect in patients with homozygous genotype (19). In later years, it was reported that R202Q (c.605G $>$ A) homozygosity was associated with the disease and could be regarded as a mutation (20). Miyoshi et al. (2008) reported the compound heterozygous composition of $\mathrm{E} 148 \mathrm{Q} / \mathrm{R} 202 \mathrm{Q}$ (c.442G $>\mathrm{C}) /(\mathrm{c} .605 \mathrm{G}>\mathrm{A}$ ) in a Japanese patient who had FMF disease (21). As shown in Table 2 , about $2 \%$ of patients have this heterozygous combination $\mathrm{E} 148 \mathrm{Q} / \mathrm{R} 202 \mathrm{Q},(\mathrm{c} .442 \mathrm{G}>\mathrm{C}) /(\mathrm{c} .605 \mathrm{G}>\mathrm{A})$ which can be the cause of the symptoms in these people. However, R202Q (c.605G $>$ A) heterozygosity has been observed in 163 healthy Japanese individuals out of 170 examined (22). This high frequency of $\mathrm{R} 202 \mathrm{Q}$ (c.605G $>\mathrm{A}$ ) heterozygous mode can be a reason that in this mode does not affect the onset of symptoms (23). This study showed that the most common age of onset of the disease is in the first and second decades of life and the most common complaints of patients are periodic fever and abdominal pain. The most common allele was M694V (c.2080A $>G$ ) in patients and the second most frequent allele was in $\mathrm{E} 148 \mathrm{Q}$ (c.442G $>$ C).

\section{Footnotes}

Authors' Contribution: H. N.F contributed to the development of the protocol, abstracted data, and prepared the manuscript and wrote the manuscript, SH. Z., MT. A. developed the original idea and analyzed data; R. SH. And SH.R. Collected the clinical data, interpreted them and revised the manuscript.

Conflict of Interests: None to declare by authors.

Funding/Support: None to declare by authors.

Informed Consent: Written consent was obtained from patients or their companions at the time of sampling. 


\section{References}

1. Sohar E, Gafni J, Pras M, Heller H. Familial Mediterranean fever. A survey of 470 cases and review of the literature. Am JMed.1967;43(2):22753. doi: 10.1016/0002-9343(67)90167-2. [PubMed: 5340644].

2. Bernot A, da Silva C, Petit JL, Cruaud C, Caloustian C, Castet V, et al. Nonfounder mutations in the MEFV gene establish this gene as the cause of familial Mediterranean fever (FMF). Hum Mol Genet. 1998;7(8):131725. doi: 10.1093/hmg/7.8.1317. [PubMed: 9668175].

3. The International FMF Consortium. Ancient missense mutations in a new member of the RoRet gene family are likely to cause familial Mediterranean fever. The International FMF Consortium. Cell. 1997;90(4):797-807. doi: 10.1016/s0092-8674(00)80539-5. [PubMed: 9288758].

4. French FMF Consortium. A candidate gene for familial Mediterranean fever. Nat Genet. 1997;17(1):25-31. doi: 10.1038/ng0997-25. [PubMed: 9288094].

5. Samuels J, Aksentijevich I, Torosyan Y, Centola M, Deng Z, Sood R, et al. Familial Mediterranean fever at the millennium. Clinical spectrum, ancient mutations, and a survey of 100 American referrals to the National Institutes of Health. Medicine (Baltimore).1998;77(4):26897. doi: 10.1097/00005792-199807000-00005. [PubMed: 9715731].

6. Cazeneuve C, Sarkisian T, Pecheux C, Dervichian M, Nedelec B, Reinert $\mathrm{P}$, et al. MEFV-Gene analysis in armenian patients with Familial Mediterranean fever: diagnostic value and unfavorable renal prognosis of the M694V homozygous genotype-genetic and therapeutic implications. Am J Hum Genet. 1999;65(1):88-97. doi: 10.1086/302459. [PubMed: 10364520]. [PubMed Central: PMC1378078].

7. Touitou I. The spectrum of familial Mediterranean fever (FMF) mutations. Eur J Hum Genet. 2001;9(7):473-83. doi: 10.1038/sj.ejhg.5200658. [PubMed: 11464238].

8. Goldfinger SE. Colchicine for familial Mediterranean fever. $N$ Engl J Med. 1972;287(25):1302. doi: 10.1056/NEJM197212212872514. [PubMed: 4636899].

9. Grateau G, Pecheux C, Cazeneuve C, Cattan D, Dervichian M, Goossens $\mathrm{M}$, et al. Clinical versus genetic diagnosis of familial Mediterranean fever. QJM. 2000;93(4):223-9. doi: 10.1093/qjmed/93.4.223. [PubMed: 10787450].

10. Akar N, Misiroglu M, Yalcinkaya F, Akar E, Cakar N, Tumer N, et al. MEFV mutations in Turkish patients suffering from familial Mediterranean fever. Hum Mutat. 2000;15(1):118-9. doi: 10.1002/(SICI)10981004(200001)15:1<118::AID-HUMU29>3.0.CO;2-5. [PubMed: 10612841].

11. Ben-Chetrit E, Urieli-Shoval S, Calko S, Abeliovich D, Matzner Y. Molecular diagnosis of FMF: Lessons from a study of 446 unrelated individuals. Clin Exp Rheumatol. 2002;20(4 Suppl 26):S25-9. [PubMed: 12371631].

12. Majeed HA, El-Khateeb M, El-Shanti H, Rabaiha ZA, Tayeh M, Najib D. The spectrum of familial Mediterranean fever gene mutations in Arabs: Report of a large series. Semin Arthritis Rheum. 2005;34(6):8138. doi: 10.1016/j.semarthrit.2005.01.010. [PubMed: 15942916].
13. Sarkisian T, Ajrapetyan H, Shahsuvaryan G. Molecular study of FMF patients in Armenia. Curr Drug Targets Inflamm Allergy. 2005;4(1):113-6. doi: 10.2174/1568010053622885. [PubMed: 15720244].

14. Esmaeili M, Bonyadi M, Rafeey M, Sakha K, Somi MH. Common MEFV mutation analysis in Iranian Azeri Turkish patients with familial Mediterranean fever. Semin Arthritis Rheum. 2008;37(5):334-8. doi: 10.1016/j.semarthrit.2007.08.005. [PubMed: 18006045].

15. Akarsu AN, Saatci U, Ozen S, Bakkaloglu A, Besbas N, Sarfarazi M. Genetic linkage study of familial Mediterranean fever (FMF) to 16p13.3 and evidence for genetic heterogeneity in the Turkish population. J Med Genet.1997;34(7):573-8. doi: 10.1136/jmg.34.7.573. [PubMed: 9222966]. [PubMed Central: PMC1050998].

16. Cazeneuve C, Ajrapetyan H, Papin S, Roudot-Thoraval F, Genevieve D, Mndjoyan E, et al. Identification of MEFV-independent modifying genetic factors for familial Mediterranean fever. Am J Hum Genet. 2000;67(5):1136-43. doi: 10.1016/S0002-9297(07)62944-9. [PubMed: 11017802]. [PubMed Central: PMC1288556].

17. Touitou I, Picot MC, Domingo C, Notarnicola C, Cattan D, Demaille $J$, et al. The MICA region determines the first modifier locus in familial Mediterranean fever. Arthritis Rheum. 2001;44(1):163-9. doi: 10.1002/1529-0131(200101)44:1<163::AID-ANR20>3.0.CO;2-Z. [PubMed: 11212154].

18. Yigit S, Karakus N, Tasliyurt T, Kaya SU, Bozkurt N, Kisacik B. Significance of MEFV gene R202Q polymorphism in Turkish familial Mediterranean fever patients. Gene. 2012;506(1):43-5. doi: 10.1016/j.gene.2012.06.074. [PubMed: 22771921].

19. Ritis K, Giaglis S, Spathari N, Micheli A, Zonios D, Tzoanopoulos $\mathrm{D}$, et al. Non-isotopic RNase cleavage assay for mutation detection in MEFV, the gene responsible for familial Mediterranean fever, in a cohort of Greek patients. Ann Rheum Dis. 2004;63(4):438-43. doi: 10.1136/ard.2003.009258. [PubMed: 15020340]. [PubMed Central: PMC1754936].

20. Giaglis S, Papadopoulos V, Kambas K, Doumas M, Tsironidou V, Rafail S, et al. MEFV alterations and population genetics analysis in a large cohort of Greek patients with familial Mediterranean fever. Clin Genet. 2007;71(5):458-67. doi: 10.1111/j.1399-0004.2007.00789.x. [PubMed: 17489852].

21. Miyoshi T, Yamashita K, Ohno T, Izumi T, Takaori-Kondo A, Sasada $\mathrm{M}$, et al. Familial Mediterranean fever gene as a possible modifier of Sweet syndrome with chronic myelogenous leukemia. Acta Haematol. 2008;120(1):57-62. doi: 10.1159/000158578. [PubMed: 18824843].

22. Yamaguchi K, Ikeda K, Ihara K, Takada H, Kusuhara K, Hara T. Lack of association between E148Q MEFV variant and Kawasaki disease. Hum Immunol. 2009;70(6):468-71. doi:10.1016/j.humimm.2008.10.017. [PubMed: 19026701].

23. Akar N, Öztürk A, Arslan C, Akar E, Taucher SC, Passalacqua C. A novel MEFV gene mutation (A511V) in a Chilean FMF patient. Egypt J Med Hum Genet. 2011;12(1):21-4. doi: 10.1016/j.ejmhg.2011.02.010. 